\title{
Grüntee-Extrakt hocheffektiv bei Genitalwarzen
}

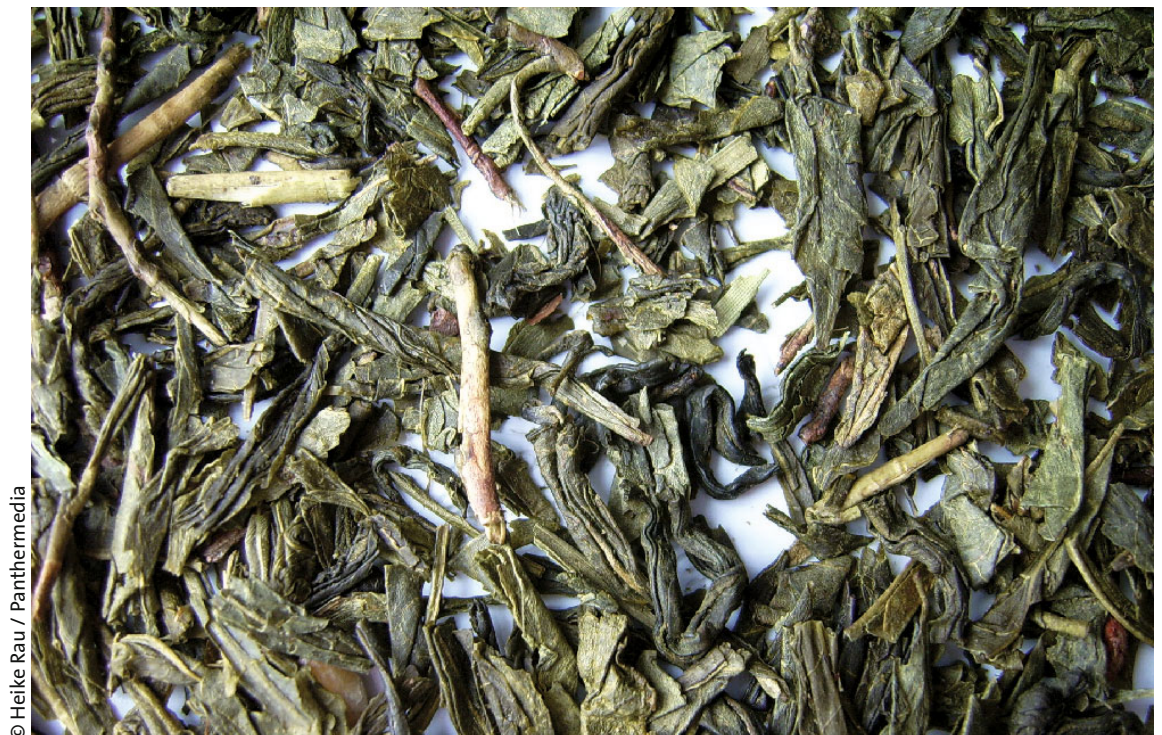

Grüner Tee ist reich an Catechinen.

- In der Therapie von Condyloma acuminata (Feig- oder Genitalwarzen) lassen sich mit Veregen ${ }^{\circledR} 10 \%$ Salbe hohe Abheilungsraten und niedrigste Rezidivraten erzielen, fasste Prof. Eggert Stockfleth, Berlin das Ergebnis der Zulassungsstudien zusammen. Der Extrakt aus Grüntee-Blättern enthält Catechine (Hauptbestandteil: Epigallocatechingallat). Der Wirkstoff hemmt die überschießende Proliferation HPV (humaner Papillomavirus)-induzierter Kerato- zyten und wirkt lokal antioxidativ, immunmodulatorisch, antiinflammatorisch und inhibiert die Virusreplikation.

Die Zusammenfassung der beiden randomisierten, doppelblinden Phase III-Studien [Tatti S et al. Br J Dermatol 2010;162:176-84] zeigte die signifikante Überlegenheit der Salbe (dreimal täglich über 16 Wochen, Follow-up 12 Wochen) gegenüber Placebo. Bei über $60 \%$ der mit Veregen ${ }^{\circledR}$ Patienten heilten die Warzen innerhalb von durchschnittlich 13 Wochen komplett $a b$ (vs. $44 \%$ ). Eine partielle Clearance mit mindestens 50\%iger Abheilung wurde bei fast $75 \%$ der Patienten erreicht (vs. $52 \%$ ). „Die Rezidivrate von 6,5\% ist verglichen mit allen anderen Therapien die mit Abstand niedrigste Rate, die wir kennen“, erklärte Stockfleth. Bei physikalisch-chirurgischen Methoden bezifferte er die Rezidivraten auf bis zu 27\% und höher. Andere topische Behandlungen zeigen ebenfalls häufige Rezidive und belasten zudem mit mehr $\mathrm{Ne}$ benwirkungen wie starken Rötungen oder Fieber. Bei Veregen ${ }^{\circledR}$ waren unerwünschte Ereignisse auf Placeboniveau (24\% vs. $21 \%$ ). Als leichte Lokalreaktion war eine milde bis mittelgradige Rötung zu beobachten, die als Zeichen der Aktivität der Behandlung gilt. Die Abbruchrate betrug 1\%.

Veregen ${ }^{\circledR}$ wird dreimal täglich dünn auf alle Warzen bis zur vollständigen Abheilung oder über maximal 16 Wochen aufgetragen. Die Salbe ist seit März 2010 in Deutschland erhältlich.

koc

Symposium „Innovative Therapieoptionen: Veregen ${ }^{\circledR}$ zur Behandlung von Feigwarzen. Ultra low dose in der Hormonersatztherapie" im Rahmen des Fortbildungskongress FOKO, Düsseldorf, 6. März 2010 Veranstalter: Solvay, Hannover

\section{Erektile Dysfunktion - Barometer der Männergesundheit}

— Die Rolle der Erektilen Dysfunktion (ED) als Barometer der Männergesundheit wird laut Dr. Graham Jackson, London, häufig unterschätzt. „Wir können die Erektile Dysfunktion nutzen, um Erkrankungen, die sonst erst Jahre später durch ein schwerwiegendes Ereignis wie einen Myokardinfarkt in Erscheinung treten würden, aufzuspüren und diesen sogar vorzubeugen", erklärte Jackson. Diese Aussage unterstützte er mit einer Kasuistik, die er auf einem Symposium im Rahmen des Kongresses der European Society of Sexual Medicine vorgestellt hat. Im Mittelpunkt stand ein 38-jähriger Patient, der seit etwa sechs Monaten unter einer ED litt. Ansonsten zeigte er keine Symptome. Die ED konnte mit Tadalafil 5 mg $\left(\right.$ Cialis $^{\circledR}$ ) einmal täglich erfolgreich behandelt werden. Außerdem entschied man sich für zusätzliche internistische Untersuchungen und kam zu einem überraschenden Befund: Bei einer CT-Angiografie konnte der Radiologe eine kurze, nicht verkalkte, stenotische Läsion in der rechten Koronararterie feststellen. Diese hätte eine relevante Verengung mit lebensbedrohlichen Auswirkungen verursachen können.

Jackson verwies außerdem auf eine Studie, an der 207 Patienten mit kardiovaskulären Erkrankungen, die ein Rehabilitationsprogramm absolvierten, und eine entsprechende Kontrollgruppe $(n=165)$ teilnahmen [Hodges et al. 2007]. 66\% der Männer mit kardiovaskulären Erkrankungen berichteten von einer ED, die im Schnitt fünf Jahre vor dem kardiovaskulären Ereignis auftrat. In der Kontrollgruppe waren dies nur $37 \%$. Das abschließende Fazit von Jackson: „Wir haben ein Zeitfenster von bis zu fünf Jahren, um kardiovaskulären Ereignissen vorzubeugen und sollten dieses unbedingt nutzen."

Zur Behandlung der ED stehen drei Therapiekonzepte zur Verfügung: Neben der Behandlung nach Bedarf mit dem länger wirksamen Tadalafil oder einem der kürzer wirksamen PDE-5-Hemmer ist mit der einmal täglichen Einnahme von 5 mg Tadalafil eine weitere wirksame und gut verträgliche Therapieoption.

Nach Informationen von

Lilly, Bad Homburg 